\section{Dr. Hardcastle, et al reply}

\section{To the Editor:}

We read with interest the report by Toussirot ${ }^{1}$ describing another case of psoriasis emerging in a patient treated with rituximab (RTX) for rheumatoid arthritis. The case described has some similarities to the woman whose case we recently reported ${ }^{2}$ in that it involves plaque psoriasis developing after a first course of RTX. However, there are a number of important differences. Whereas our patient was seronegative with a positive family history and previous episode of guttate psoriasis, the patient described here was seropositive, with no known history or family history of psoriasis. The lesion in this case appeared in a typical location (the scalp), whereas our patient had an atypical acral distribution of lesions. The lag time between treatment and onset of skin lesions was also shorter in our patient ( 1 month) than the 5-month interval described by Toussirot.

Our patient's subsequent course is interesting. Her skin lesions took 6 months to resolve. After 11 months she requested rechallenge with RTX because her joints were in flare. A severe skin reaction recurred 6 weeks after RTX treatment. The lesions were similar in character and distribution (hands, face, and feet) to the first reaction but were more intense and took longer to settle.

Given the number of previous case reports, it does seem likely that RTX has a role in inducing psoriasis or psoriasiform lesions in some patients, in common with other biologics such as anti-tumor necrosis factor agents $^{3}$. This effect has not yet been observed in registry-based studies ${ }^{4}$, suggesting that the overall incidence is small. As the authors point out, the particularly interesting thing about this case is that the patient had previously received a number of other biologic agents, in contrast to our patient, who was biologic-naive. Whereas it could be argued that our patient had a predisposition to develop psoriasis given an appropriate trigger, this case suggests that both patient-related and drug-specific factors are implicated in these reactions. In support of this, other patients with either preexisting psoriasis or psoriatic arthritis seem to be able to tolerate RTX without any dermatological deterioration ${ }^{5}$, and as the author states, have sometimes been reported to improve. While underlying mechanisms behind these varied reactions remain unclear, once elucidated they have the potential to further our understanding of both psoriasis and the complex actions of biologic agents such as RTX.

SARAH A. HARDCASTLE, MBChB, BSc, MRCP, Clinical Research Fellow, Musculoskeletal Research Unit, University of Bristol, Bristol; SAM

GIBBS, MA, MB, BChir, FRCP, Consultant Dermatologist, Department of Dermatology, The Great Western Hospital, Swindon; LYN WILLIAMSON, BM, BCh, BA, FRCP, Consultant Rheumatologist, Department of Rheumatology, The Great Western Hospital, Swindon, UK. Address correspondence to Dr. S. Hardcastle, Academic Rheumatology, Musculoskeletal Research Unit, Avon Orthopaedic Centre, Southmead Hospital, Bristol BS10 5NB, UK.

E-mail: Sarah.Hardcastle@bristol.ac.uk

\section{REFERENCES}

1. Toussirot E. New onset of psoriasis in a patient with rheumatoid arthritis treated with rituximab [letter]. J Rheumatol 2013; 40:1230-1.

2. Hardcastle SA, Gibbs S, Williamson L. Atypical psoriasis following rituximab for rheumatoid arthritis. J Rheumatol 2012;39:1303-4.

3. Grinblat B, Scheinberg M. The enigmatic development of psoriasis and psoriasiform lesions during anti-TNF therapy: A review. Semin Arthritis Rheum 2008;37:251-5.

4. Thomas L, Canoui-Poitrine F, Gottenberg JE, Economu-Dubosc A, Medkour F, Chevalier X, et al. Incidence of new-onset and flare of preexisting psoriasis during rituximab therapy for rheumatoid arthritis: Data from the French AIR registry. J Rheumatol 2012;39:893-8.

5. Jimenez-Boj E, Stamm TA, Sadlonova M, Rovensky J, Raffayová $\mathrm{H}$, Leeb B, et al. Rituximab in psoriatic arthritis: An exploratory evaluation. Ann Rheum Dis 2012;71:1868-71.

J Rheumatol 2013;40:7; doi:10.3899/jrheum.130279 